\title{
EDITORIAL
}

\section{The future of networks based on the vision of the SDN}

Jorge Gómez Gómez - PhD

Editor Revista Ingeniera e Innovación

Traditionally computer networks as we know them are based on a physical infrastructure called hardware that brings with it pre-determined software developed by each of the manufacturers. In reality, networks face a great challenge as the needs of both mobile and fixed customers grow. These needs are oriented to immediate access to all types of content, which obviously generates implications in the acquisition of bandwidth and additional purchase of hardware, to offer better performance to the network infrastructure. It would seem to be a simple solution, but in many cases, despite the addition of new equipment and more bandwidth, can not necessarily solve the problem.

To put this into context, let's think of a situation where traffic grows exponentially in a given time and the network must be able to respond to the needs of all users:

Usually every 29th of November, Black Friday is celebrated all over the world. As we all know, there is a world of promotions in all physical and virtual stores. That day is one of the largest in the network. Which implies that for an online wholesale store, this must be able to meet all the requests of customers who want to buy?. This is good news for the store but it is a huge workload for the network infrastructure that will support this traffic. As noted above, one solution is to expand bandwidth and acquire more hardware for the data center. It's not necessarily an optimal solution, because it involves financial expenses, energy consumption by devices, and the tedious work of configuring network hardware.

Faced with the previous challenge, a solution is being orchestrated that allows networks to be managed dynamically, depending on the needs of the applications. A solution that is glimpsed are the SDN (Software Defined Network). This type of networks is based on three main layers: the application layer, control and infrastructure. In the application layer reside the applications used by organizations. These applications, in addition to those used by end users, also include load balancers, firewalls, intrusion detectors, among others. The control layer represents the centralized controller of the SDN. In essence, this controller is located on a server that manages network traffic and policies. Finally, the infrastructure layer is formed by the physical switches of the network. The layers communicate with each other through the north and south interfaces. In the case of the application layer, it communicates via the northern interface. The switches do this with the south interface using the OpenFlow protocol, although in practice there are several types of protocols to connect this interface, this is one of the most used.

The previous architecture of the SDN, allows greater flexibility to networks, so that makes them more dynamic and respond to the needs of applications. Many manufacturers of network devices are experimentally including the OpenFlow protocol in the hardware, which makes it easier for administrators to administer through a server to the network. Companies such as Google, Facebook and others are already testing SDN for their network infrastructure to respond to the traffic demand of their users. 
Like all technology, SDNs have advantages and disadvantages, as shown below:

Advantages:

- An administrator can change the network rules at any time.

- An administrator only needs one centralized controller, to distribute policies to the different connected switches, instead of configuring them individually.

- Traffic can be monitored and security policies implemented.

- Enables hardware to be virtualized

Disadvantages:

The main disadvantage seen in the SDN is associated with security, because the controller is centralized and an attack on the server would generate a single point of failure that would cause the network to stop working. While this is a strong trend towards where networks are oriented and which could improve efficiency, there is still a long way to go because there is no standardization on this technology. 